\title{
PICARD-VESSIOT THEORY OF PARTIAL DIFFERENTIAL FIELDS
}

\author{
E. R. KOLCHIN
}

Introduction. ${ }^{1}$ The purpose of this note is to extend to partial differential fields the Picard-Vessiot theory of homogeneous linear ordinary differential equations. ${ }^{2}$ Although this can be done by extending the procedure of $\mathrm{PV}$ to partial differential fields, it is perhaps not without interest to see how the theory in the partial case can be deduced from the ordinary theory, and the latter is what is done below.

If $\mathcal{F}$ is an ordinary differential field of characteristic 0 with algebraically closed field of constants and $G$ is an extension of $\mathcal{F}$ with the same field of constants, then (PV, $\$ 17) G$ is a Picard-Vessiot extension of $\mathcal{F}$ if there exists a homogeneous linear differential equation $y^{(n)}+p_{1} y^{(n-1)}+\cdots+p_{n} y=0\left(\right.$ each $\left.p_{i} \in \mathcal{F}\right)$ with a fundamental system of solutions $\eta_{1}, \cdots, \eta_{n}$ such that $G=\mathcal{F}\left\langle\eta_{1}, \cdots, \eta_{n}\right\rangle$. Since $p_{i}$ is then plus or minus the quotient of two determinants $W_{i} / W_{0}$, where $W_{l}=\operatorname{det}\left(\eta_{k}^{(j)}\right)_{0 \leqq j \leqq n, j \neq n-l ; \leqq k \leqq n}$, it is the same thing to require that $G$ contain elements $\eta_{1}, \cdots, \eta_{n}$ linearly independent over constants such that $G=\mathcal{F}\left\langle\eta_{1}, \cdots, \eta_{n}\right\rangle$ and such that each $W_{i} / W_{0}$ belongs to $\mathcal{F}$. It is this property which is generalized below to define "Picard-Vessiot extension" in the case of partial differential fields.

1. Linear dependence over constants. Let $G$ be a partial differential field of characteristic 0 with $m$ derivations $\delta_{1}, \cdots, \delta_{m}$; denote the field of constants of $G$ by $C$. Let $u_{1}, \cdots, u_{m}$ be elements of some extension of $G$ which are differentially algebraically independent over $G$, and consider the partial differential field $G\left\langle u_{1}, \cdots, u_{m}\right\rangle$. The operator $D=\sum u_{i} \delta_{i}$ is then a derivation of $G\left\langle u_{1}, \cdots, u_{m}\right\rangle$ and therefore induces in $G\left\langle u_{1}, \cdots, u_{m}\right\rangle$ a structure of an ordinary differential field; we denote the ordinary differential field so defined by $G_{D}$. The field of constants of $G_{D}$ is $C_{\text {; indeed if we order the expressions }}$ $\delta_{1}^{i_{1}} \cdots \delta_{m}^{t_{m}} u_{j}$ lexicographically and if $\delta_{1}^{a_{1}} \cdots \delta_{m}^{a_{m}} u_{b}$ is the highest such expression effectively present in some element $\phi \in G_{D}(\phi \notin G)$, then $\delta_{1}^{a_{1}+1} \delta_{2}^{a_{2}} \cdots \delta_{m}^{a_{m}} u_{b}$ is effectively present in $D \phi$, so that $D \phi \neq 0$.

Let $y_{1}, \cdots, y_{n}$ be indeterminates, and let $\theta_{1}, \cdots, \theta_{n}$ denote

Received by the editors November 20, 1951.

1 The research on which this paper is based was done in connection with a contract with the Office of Naval Research.

${ }^{2}$ It is assumed that the reader is familiar with my paper on this subject in Ann. of Math. vol. 49 (1948) pp. 1-42, which will be referred to as PV. 
differential operators of the form $\delta_{1}^{i_{1}} \cdots \delta_{m}^{t_{m}}$, where each $i_{\nu}$ is an integer not less than 0 . Then we may form the determinant $W_{\theta_{1} \cdots \theta_{n}}=W_{\theta_{1} \cdots \theta_{n}}\left(y_{1}, \cdots, y_{n}\right)=\operatorname{det}\left(\theta_{i} y_{j}\right)_{1 \leqq i \leqq n, 1 \leqq j \leqq n}$. The wronskian $\operatorname{det}\left(D^{i} y_{j}\right)_{0 \leqq i \leqq n-1,1 \leqq j \leqq n}$ obviously can be expressed as a linear combination of determinants $W_{\theta_{1} \cdots \theta_{n}}$ in which each $\theta_{i}$ is of order not greater than $n-1$.

Lemma 1. Let $\eta_{1}, \cdots, \eta_{n} \in G$. If $\eta_{1}, \cdots, \eta_{n}$ are linearly dependent over $\mathcal{C}$, then $W_{\theta_{1}} \ldots \theta_{n}\left(\eta_{1}, \cdots, \eta_{n}\right)=0$ for every choice of $\theta_{1}, \cdots, \theta_{n}$. Conversely if $W_{\theta_{1}} \ldots \theta_{n}\left(\eta_{1}, \cdots, \eta_{n}\right)=0$ for every choice of $\theta_{1}, \cdots, \theta_{n}$ of order not greater than $n-1$, then $\eta_{1}, \cdots, \eta_{n}$ are linearly dependent over $C$.

Proof. If $\sum c_{j} \eta_{j}=0\left(c_{1}, \cdots, c_{n} \in \mathcal{C}\right.$, not all 0$)$, then $\sum c_{j} \theta_{i} \eta_{j}$ $=0(1 \leqq i \leqq n)$ for all choices of $\theta_{1}, \cdots, \theta_{n}$, whence $W_{\theta_{1}} \ldots \theta_{n}\left(\eta_{1}, \cdots, \eta_{n}\right)$ $=0$. Conversely if $W_{\theta_{1}} \ldots \theta_{n}\left(\eta_{1}, \cdots, \eta_{n}\right)=0$ for all choices of $\theta_{1}, \cdots$, $\theta_{n}$ of order not greater than $n-1$ then, by a remark made above, the wronskian $\operatorname{det}\left(D^{i} \eta_{j}\right)=0$; since $G_{D}$ is an ordinary differential field it is known ( $\mathrm{PV}, \S 14)$ that this implies that $\eta_{1}, \cdots, \eta_{n}$ are linearly dependent over the field of constants of $G_{D}$, that is over $C$.

Since the coefficients in $W_{\theta_{1}} \cdots \theta_{n}$ are integers, the lemma shows that $\eta_{1}, \cdots, \eta_{n}$ are linearly dependent (or independent) over $C$ if and only if they are so over the field of constants of any partial differential field containing $\eta_{1}, \cdots, \eta_{n}$. Because of this we may speak simply of linear dependence (or independence) over constants.

2. Two lemmas. We continue with $G, \mathcal{C}, G_{D}$ as in $\$ 1$.

Lemma 2. Let $\eta_{1}, \cdots, \eta_{n} \in G$ be linearly independent over $\mathcal{C}$, so that $W_{\theta_{01} \cdots \theta_{0 n}}\left(\eta_{1}, \cdots, \eta_{n}\right) \neq 0$ for some choice of operators $\theta_{01}, \cdots, \theta_{0 n}$ of order not greater than $n-1$; let $\mathcal{F}$ be a partial differential subfield of $G$ which contains

$$
W_{\theta_{1}} \ldots \theta_{n}\left(\eta_{1}, \cdots, \eta_{n}\right) / W_{\theta_{01} \cdots \theta_{0 n}}\left(\eta_{1}, \cdots, \eta_{n}\right)
$$

for every choice of $\theta_{1}, \cdots, \theta_{n}$ of order not greater than $n$. Then $\mathcal{F}$ contains (1) for every choice of $\theta_{1}, \cdots, \theta_{n}$ regardless of order.

Proof. Each $\eta_{j}$ is a zero of the partial differential polynomial

$$
W_{\theta_{0} \theta_{1} \cdots \theta_{n}}\left(y, \eta_{1}, \cdots, \eta_{n}\right) / W_{\theta_{01} \cdots \theta_{0 n}}\left(\eta_{1}, \cdots, \eta_{n}\right)
$$

for every choice of $\theta_{0}, \theta_{1}, \cdots, \theta_{n}$. When $\theta_{0}, \theta_{1}, \cdots, \theta_{n}$ are all of order not greater than $n$, then, by the hypothesis of the lemma, each coefficient in (2) is in $\mathcal{F}$. Therefore if $\sigma$ is any isomorphism of $G$ over $\mathcal{F}(\mathrm{PV}, \S 12)$, then $\sigma \eta_{j}$ is a zero of (2) whenever $\theta_{0}, \theta_{1}, \cdots, \theta_{n}$ are of order not greater than $n$. It follows from Lemma 1 that $\sigma \eta_{j}, \eta_{1}, \cdots, \eta_{n}$ 
are linearly dependent over constants, that is, $\sigma \eta_{j}=\sum k_{i j} \eta_{i}$ $(1 \leqq j \leqq n)$, where the elements $k_{i j}$ are constants in an extension of $G$. Therefore $\sigma W_{\theta_{1}} \ldots \theta_{n}\left(\eta_{1}, \cdots, \eta_{n}\right)=\operatorname{det}\left(k_{i j}\right) \cdot W_{\theta_{1}} \ldots \theta_{n}\left(\eta_{1}, \cdots, \eta_{n}\right)$, so that every expression (1) is invariant under every isomorphism $\sigma$ of $G$ over $\mathcal{F}$, whence (PV, $\$ 12$ ) every expression (1) belongs to $\mathcal{F}$.

If $\mathcal{F}$ is any partial differential subfield of $G$, then $\mathcal{F}\left\langle u_{1}, \cdots, u_{m}\right\rangle$ is a partial differential subfield of $G\left\langle u_{1}, \cdots, u_{m}\right\rangle$; it is clear that we may also regard $\mathcal{F}\left\langle u_{1}, \cdots, u_{m}\right\rangle$ as an ordinary differential subfield of $G_{D}$, which we shall denote by $\mathcal{F}_{D}$. If $\eta_{1}, \cdots, \eta_{n}$ are any elements of $G$, then we naturally denote by $\mathcal{F}_{D}\left\langle\eta_{1}, \cdots, \eta_{n}\right\rangle$ the ordinary differential field extension, which set-theoretically coincides with $\mathcal{F}_{D}\left(\left(D^{i} \eta_{j}\right)_{0 \leqq i<\infty, 1 \leqq j \leqq n}\right)$.

Lemмa 3. Under the hypotheses of Lemma 2 and the assumption that $G=\mathcal{F}\left\langle\eta_{1}, \cdots, \eta_{n}\right\rangle$ we have $G_{D}=\mathcal{F}_{D}\left\langle\eta_{1}, \cdots, \eta_{n}\right\rangle$.

Proof. We must show for every $j$ and for every operator $\theta$ $=\delta_{1}^{i_{1}} \cdots \delta_{m}^{l_{m}}$ that $\theta \eta_{j} \in \mathcal{F}_{D}\left\langle\eta_{1}, \cdots, \eta_{n}\right\rangle$. Now $\left(\eta_{1}, \cdots, \eta_{n}\right)$ is a fundamental system of solutions of the homogeneous linear ordinary differential equation

$$
W_{\theta_{01}} \cdots \theta_{0 n}\left(\eta_{1}, \cdots, \eta_{n}\right)^{-1} W\left(y, \eta_{1}, \cdots, \eta_{n}\right)=0,
$$

where $W\left(y, \eta_{1}, \cdots, \eta_{n}\right)$ is the wronskian determinant (with respect to the derivation $D$ ) of $y, \eta_{1}, \cdots, \eta_{n} ;(3)$ is of order $n$ and obviously has its coefficients in $\mathcal{F}_{D}$. Let

$$
D^{k} y+v_{1} D^{k-1} y+\cdots+v_{k} y=0
$$

be the homogeneous linear ordinary differential equation of lowest order with coefficients in $\mathcal{F}_{D}$ and with solution $\eta_{1}$. Every solution of (4) is a solution of (3), so there exist $k$ linear combinations (with constant coefficients) $\zeta_{1}=\eta_{1}, \zeta_{2}, \cdots, \zeta_{k}$ of $\eta_{1}, \cdots, \eta_{n}$ such that $\left(\zeta_{1}, \cdots, \zeta_{k}\right)$ is a fundamental system of solutions of (4). Since $\zeta_{1}, \cdots, \zeta_{k}$ are linearly independent over constants, there exist operators $\lambda_{1}, \cdots, \lambda_{k}$ of order not greater than $k-1$ such that $W_{\lambda_{1} \cdots \lambda_{k}}\left(\zeta_{1}, \cdots, \zeta_{k}\right) \neq 0$.

Every isomorphism of $G$ over $\mathcal{F}$ obviously maps $\zeta_{1}, \cdots, \zeta_{k}$ into $k$ solutions of (4) linearly independent over constants, and therefore performs a linear substitution (with constant coefficients and nonzero determinant) on $\zeta_{1}, \cdots, \zeta_{k}$; it follows that every such isomorphism leaves invariant the element

$$
W_{\theta_{1}} \cdots \theta_{k}\left(\zeta_{1}, \cdots, \zeta_{k}\right) / W_{\lambda_{1}} \cdots \lambda_{k}\left(\zeta_{1}, \cdots, \zeta_{k}\right)
$$

for every choice of $\theta_{1}, \cdots, \theta_{k}$, so that every such element belongs to 
7. Thus for each $\theta$ the equation

$$
W_{\theta \lambda_{1}} \cdots \lambda_{k}\left(y, \zeta_{1}, \cdots, \zeta_{k}\right) / W_{\lambda_{1} \cdots \lambda_{k}}\left(\zeta_{1}, \cdots, \zeta_{k}\right)=0,
$$

which is satisfied by $\zeta_{1}=\eta_{1}$, has its coefficients in $\mathcal{F}$. It follows that for each operator $\theta$ there exist elements $a_{\theta 1}, \cdots, a_{\theta k} \in \mathcal{F}$ such that

$$
\theta \eta_{1}=\sum_{j=1}^{k} a_{\theta j} \lambda_{j} \eta_{1} .
$$

Consequently for each integer $i \geqq 0$ there exist elements $v_{i 1}, \cdots, v_{i k}$ $\in \mathcal{F}_{D}$ such that

$$
D^{i} \eta_{1}=\sum_{j=1}^{k} v_{i j} \lambda_{j} \eta_{1} .
$$

If the determinant of $\left(v_{i j}\right)_{0 \leqq i \leqq k-1,1 \leqq j \leqq k}$ were 0 , then $\eta_{1}, D \eta_{1}, \cdots$, $D^{k-1} \eta_{1}$ would be linearly dependent over $\mathcal{F}_{D}$, that is, $\eta_{1}$ would satisfy a homogeneous linear differential equation over $\mathcal{F}_{D}$ of order less than $k$. Therefore this determinant is nonzero and we can express each $\lambda_{j} \eta_{1}$ linearly over $\mathcal{F}_{D}$ in terms of $\eta_{1}, D \eta_{1}, \cdots, D^{k-1} \eta_{1}$, so that $\lambda_{j} \eta_{1}$ $\in \mathcal{F}_{D}\left\langle\eta_{1}\right\rangle$, whence, by $(5), \theta \eta_{1} \in \mathcal{F}_{D}\left\langle\eta_{1}\right\rangle$ for every differential operator $\theta$. Similarly $\theta \eta_{j} \in \mathcal{F}_{D}\left\langle\eta_{j}\right\rangle$ for each $j$.

3. Picard-Vessiot theory. Let $\mathcal{F}$ be a partial differential field of characteristic 0 with $m$ derivations $\delta_{1}, \cdots, \delta_{m}$ and with algebraically closed field of constants $\mathcal{C}$, and let $G$ be an extension of $\mathcal{F}$. We shall call $G$ a Picard-Vessiot extension of $\mathcal{F}$ if: (1) the field of constants of $G$ is $C_{\text {; }}$ (2) $G$ contains a finite family of elements $\left(\eta_{1}, \cdots, \eta_{n}\right)$ which is linearly independent over constants, has the property that $\mathcal{F}\left\langle\eta_{1}, \cdots, \eta_{n}\right\rangle=G$, and has the property that

$$
W_{\theta_{1}} \cdots \theta_{n}\left(\eta_{1}, \cdots, \eta_{n}\right) / W_{\theta_{01} \cdots \theta_{0 n}}\left(\eta_{1}, \cdots, \eta_{n}\right) \in \mathcal{F}
$$

for all choices of $\theta_{1}, \cdots, \theta_{n}$ of order not greater than $n$ and some fixed $\theta_{01}, \cdots, \theta_{0 n}$ such that $W_{\theta_{01} \cdots \theta_{0 n}}\left(\eta_{1}, \cdots, \eta_{n}\right) \neq 0$. As seen in the introduction, for "partial" differential fields with one derivation (case $m=1$ ), that is, for ordinary differential fields, this coincides with the concept of Picard-Vessiot extension already known.

Let $G$ be such a Picard-Vessiot extension of $\mathcal{F}_{\text {, and let }} \mathcal{F}_{D}$ and $G_{D}$ be as before. By Lemma 3, $G_{D}$ is an ordinary Picard-Vessiot extension of $\mathcal{F}_{D}$. It follows that the equations

$$
\sigma \eta_{j}=\sum_{i=1}^{n} s_{i j} \eta_{i} \quad(1 \leqq j \leqq n)
$$


establish an isomorphism between the group of all automorphisms $\sigma$ of $G_{D}$ over $\mathcal{F}_{D}$ and a certain algebraic group (B) of matrices $\left(s_{i j}\right)$ over C. By (6) we see that $\sigma G=G$; also, for each $\alpha \in G$ we have

$$
\sum u_{i}\left(\sigma \delta_{i} \alpha-\delta_{i} \sigma \alpha\right)=\sigma \sum u_{i} \delta_{i} \alpha-\sum u_{i} \delta_{i} \sigma \alpha=\sigma D \alpha-D \sigma \alpha=0
$$

whence, since $u_{1}, \cdots, u_{m}$ are differentially algebraically independent over $G, \sigma \delta_{i} \alpha=\delta_{i} \sigma \alpha(1 \leqq i \leqq m)$. It follows that the restriction to $G$ of every automorphism of $G_{D}$ over $\mathcal{F}_{D}$ is an automorphism of $G$ over F.

Conversely if we start with an automorphism of $G$ over $\mathcal{F}$, it can be extended to a unique automorphism of $G\left\langle u_{1}, \cdots, u_{m}\right\rangle$ over $\mathcal{F}\left\langle u_{1}, \cdots, u_{m}\right\rangle$, which is a fortiori an automorphism of $G_{D}$ over $\mathcal{F}_{D}$.

Summarizing these remarks we have the following theorem.

Theorem 1. If $G$ is a Picard-Vessiot extension of $\mathcal{F}$, then $G_{D}$ is a Picard-Vessiot extension of $\mathcal{F}_{D}$, the mapping which to each automorphism of $G_{D}$ over $\mathcal{F}_{D}$ assigns its restriction to $G$ is an isomorphism of the group of all automorphisms of $G_{D}$ over $\mathcal{F}_{D}$ onto the group of all automorphisms of $G$ over $\mathcal{F}$, and the equations (6) establish an isomorphism of each of these groups with an algebraic matric group over $\mathcal{C}$.

By virtue of this theorem we identify the three groups mentioned therein and denote them all by $\left(5\right.$. If $\mathcal{F}_{1}$ is a partial differential field between $\mathcal{F}$ and $G$ and if we denote the group of all automorphisms of $G$ over $\mathcal{F}_{1}$ by $\mathcal{S}\left(\mathcal{F}_{1}\right)$, then $B\left(\mathcal{F}_{1}\right)$ is also (identified with) the group of all automorphisms of $G_{D}$ over $\mathcal{F}_{1 D}$ (where $\mathcal{F}_{1 D}$ is $\mathcal{F}_{1}\left\langle u_{1}, \cdots, u_{m}\right\rangle$ considered as an ordinary differential field with derivation $D$ ); an element in $G$ but not in $\mathcal{F}_{1}$ fails to belong to $\mathcal{F}_{1 D}$ and therefore (because $G_{D}$ is normal over $\left.\mathcal{F}_{D}\right)$ is not an invariant of $\mathscr{G}\left(\mathcal{F}_{1}\right)$. Consequently $G$ is normal over $\mathcal{F}(\mathrm{PV}, \S 16)$. Of course $\$\left(\mathcal{F}_{1}\right)$ is an algebraic subgroup of $B$; the fact that every algebraic subgroup of $B$ is some $\mathcal{S}\left(\mathcal{F}_{1}\right)$ with $\mathcal{F}_{1}$ a partial differential field between $\mathcal{F}$ and $G$ can be proved exactly as in the ordinary case (PV, 119$)$. Thus $\mathscr{F}_{1} \rightarrow\left(S\left(\mathcal{F}_{1}\right)\right.$ is a one-to-one mapping of the set of all partial differential fields between $\mathcal{F}$ and $G$ onto the set of all algebraic subgroups of $B$. Since there is a similar one-to-one mapping of the set of all ordinary differential fields between $\mathcal{F}_{D}$ and $G_{D}$ onto the set of all algebraic subgroups of $B$, we see that every differential field between $\mathcal{F}_{D}$ and $G_{D}$ is the form $\mathcal{F}_{1 D}$, where $\mathcal{F}_{1}$ is a partial differential field between $\mathcal{F}$ and $G$. The degree of transcendence of $G$ over $\mathcal{F}_{1}$ obviously equals that of $G_{D}$ over $\mathcal{F}_{1 D}$, which by the ordinary Picard-Vessiot theory (PV, $\S 20)$ equals the dimension of the algebraic group $B\left(\mathcal{F}_{1}\right)$. Thus we have the following theorem. 
Theorem 2. Let $G$ be a Picard-Vessiot extension of $\mathcal{F}$. Then $\mathcal{F}_{1} \rightarrow \mathfrak{B}\left(\mathcal{F}_{1}\right)$ is a one-to-one mapping of the set of all partial differential fields between $\mathcal{F}$ and $G$ onto the set of all algebraic subgroups of $\mathbb{( S ,}$ such that the transcendence degree of $G$ over $\mathcal{F}_{1}$ equals the dimension of $\mathrm{S}\left(\mathcal{F}_{1}\right)$. Also, $\mathcal{F}_{1} \rightarrow \mathcal{F}_{1 D}$ is a one-to-one mapping of the set of all partial differential fields between $\mathcal{F}$ and $G$ onto the set of all ordinary differential fields between $\mathcal{F}_{D}$ and $G_{D}$.

Continuing, let $\mathcal{F}_{1}$ be a partial differential field between $\mathcal{F}$ and $G$. If $\sigma_{1}$ is an isomorphism over $\mathcal{F}$ of $\mathcal{F}_{1}$ into $G$, then $\sigma_{1}$ can obviously be extended to an isomorphism over $\mathcal{F}\left\langle u_{1}, \cdots, u_{m}\right\rangle$ of $\mathcal{f}_{1}\left\langle u_{1}, \cdots, u_{m}\right\rangle$ into $G\left\langle u_{1}, \cdots, u_{m}\right\rangle$, which is of course also an isomorphism over $\mathcal{F}_{D}$ of $\mathcal{F}_{1 D}$ into $G_{D}$. By the ordinary Picard-Vessiot theory (PV, 119 , lemma) this can in turn be extended to an automorphism of $G_{D}$, and by Theorem 1 the restriction of this to $G$ is an automorphism of $G$. This proves the following theorem.

Theorem 3. If $G$ is a Picard-Vessiot extension of $\mathcal{F}$, and $\mathcal{F}_{1}$ is a partial differential field between $\mathcal{F}$ and $G$, then every isomorphism over $\mathcal{F}$ of $\mathcal{F}_{1}$ into $G$ can be extended to an automorphism of $G$.

Exactly as in the ordinary case (PV, $\$ 16$, Theorem $2, \S 18$, Theorem 2) we have the following

CoRollary. $\mathfrak{S}\left(\mathcal{F}_{1}\right)$ is a normal subgroup if and only if $\sigma \mathcal{F}_{1}=\mathcal{F}_{1}$ for every automorphism $\sigma$ of $G$ over $\mathcal{F}$; when this is the case the mapping which to each such $\sigma$ assigns its restriction to $\mathcal{F}_{1}$ is a homomorphism with kernel $B\left(\mathcal{F}_{1}\right)$ of $B$ onto the group of all automorphisms of $\mathcal{F}_{1}$ over $\mathcal{F}$.

4. Liouvillian extensions. Suppose as before that the field of constants $\mathcal{C}$ of $\mathcal{F}$ is algebraically closed.

Let $\alpha$ be an element of an extension of $\mathcal{F}$ such that the field of constants of $\mathcal{F}\langle\alpha\rangle$ is $\mathcal{C}, \alpha \notin \mathcal{F}$, and $\delta_{i} \alpha \in \mathcal{F}(1 \leqq i \leqq m)$. Now $W_{1 \delta_{i}}(1, \alpha)$ $=\delta_{i} \alpha \in \mathcal{F}$, and these are not all zero (for otherwise $\alpha$ would be a constant and belong to $\mathcal{F})$; also, $W_{1, \delta_{i} \delta_{j}}(1, \alpha)=\delta_{i} \delta_{j} \alpha \in \mathcal{F}$, and $W_{\theta_{1} \theta_{2}}(1, \alpha)=0$ if both $\theta_{1}, \theta_{2}$ have order not less than 1 . Therefore $\mathcal{F}\langle\alpha\rangle=\mathcal{F}\langle 1, \alpha\rangle$ is a Picard-Vessiot extension of $\mathcal{F}$. Since $\mathcal{F}\langle\alpha\rangle_{D}=\mathcal{F}_{D}\langle\alpha\rangle$ and $D \alpha=\sum u_{i} \delta_{i} \alpha \in \mathcal{F}_{D}$ we see $(\mathrm{PV}, \S 23)$ that we may identify the group of all automorphisms of $\mathcal{F}\langle\alpha\rangle$ over $\mathcal{F}$ with the abelian algebraic group of all matrices of degree two of the form

$$
\left(\begin{array}{ll}
1 & c \\
0 & 1
\end{array}\right), \quad c \in \mathcal{C}
$$

Starting afresh, let $\alpha$ be an element of an extension of $\mathcal{F}$ such that 
the field of constants of $\mathcal{F}\langle\alpha\rangle$ is $\mathcal{C}, \alpha \notin \mathcal{F}$, and $\alpha^{-1} \delta_{i} \alpha \in \mathcal{F}(1 \leqq i \leqq m)$. We have $W_{1}(\alpha)=\alpha, W_{\delta_{i}}(\alpha)=\delta_{i} \alpha$ so that $W_{\delta_{i}}(\alpha) / W_{1}(\alpha)=\alpha^{-1} \delta_{i} \alpha \in \mathcal{F}$. Therefore $\mathcal{F}\langle\alpha\rangle$ is a Picard-Vessiot extension of $\mathcal{F}$. Since $\alpha^{-1} D \alpha$ $=\alpha^{-1} \sum u_{i} \delta_{i} \alpha \in \mathcal{F}_{D}$, we see $(\mathrm{PV}, \S 23)$ that we may identify the group of all automorphisms of $\mathcal{F}\langle\alpha\rangle$ over $\mathcal{F}$ either (if $\alpha$ is transcendental over $\mathcal{F}$ ) with the group of all matrices of degree one of the form (c), with $c \notin C, c \neq 0$, or (if $\alpha$ is algebraic of degree $h$ over $\mathcal{F}$ ) with the group of all such matrices with $c$ an $h$ th root of unity (in which case we have $\alpha^{h} \in \mathcal{F}$ ).

Let $\mathfrak{H C}$ be an extension of $\mathcal{F}$. We shall call $\mathfrak{H C}$ a liouvillian extension of $\mathcal{F}$ if the field of constants of $\mathfrak{F}$ is $\mathcal{C}$ and if $\mathcal{H}$ contains a finite sequence of elements $\alpha_{1}, \cdots, \alpha_{r}$ such that $\mathcal{F}\left\langle\alpha_{1}, \cdots, \alpha_{r}\right\rangle=\mathfrak{F}$ and for each $j(1 \leqq j \leqq r)$ either $\alpha_{j}$ is algebraic over $\mathcal{F}\left\langle\alpha_{1}, \cdots, \alpha_{j-1}\right\rangle$, or $\delta_{i} \alpha_{j} \in \mathcal{F}\left\langle\alpha_{1}, \cdots, \alpha_{j-1}\right\rangle(1 \leqq i \leqq m)$, or $\alpha_{j}^{-1} \delta_{i} \alpha_{j} \in \mathcal{F}\left\langle\alpha_{1}, \cdots, \alpha_{j-1}\right\rangle(1 \leqq i$ $\leqq m)$. The concept of liouvillian extension, already familiar for ordinary differential fields, is hereby extended to partial differential fields.

Suppose that, as above, $\mathfrak{H C}$ is a liouvillian extension of $\mathcal{F}$ with $\mathfrak{F}=\mathcal{F}\left\langle\alpha_{1}, \cdots, \alpha_{r}\right\rangle$. It follows that $\mathfrak{H}_{D}$ is a liouvillian extension of $\mathcal{F}_{D}$ with $\mathfrak{F}_{D}=\mathcal{F}_{D}\left\langle\alpha_{1}, \cdots, \alpha_{r}\right\rangle$.

If a Picard-Vessiot extension $G$ of $\mathcal{F}$ is contained in a liouvillian extension $\mathfrak{H}$ of $\mathcal{F}$, it follows from the above that $G_{D}$ is an ordinary Picard-Vessiot extension of $\mathcal{F}_{D}$ contained in the (ordinary) liouvillian extension $\mathfrak{H}_{D}$ of $\mathcal{F}_{D}$. By the ordinary theory $(\mathrm{PV}, \S 25)$ this implies that the group (f) of all automorphisms of $G$ over $\mathcal{F}$ has a solvable component of the identity (5).

Conversely, suppose the component of the identity $\left(55^{\circ}\right.$ is solvable. If $\mathcal{F}^{0}$ is the partial differential field between $\mathcal{F}$ and $G$ such that (530 $=\left(\mathfrak{S}\left(\mathfrak{F}^{0}\right)\right.$, then $G$ is a Picard-Vessiot extension of $\mathcal{F}^{0}, \mathcal{F}^{0}$ is an algebraic extension of $\mathcal{F}$, and the group of all automorphisms of $G$ over $\mathrm{F}^{0}$ is (5) $^{\circ}$. Since (5) $^{\circ}$ is solvable we may assume (PV, $\$ 7$, Theorem 1) that $\left(5^{\circ}\right.$ (identified with an algebraic matric group by means of the equations (6)) is in triangular form, that is, for each $\sigma \in\left(S^{0}\right.$ we have:

$$
\sigma \eta_{j}=\sum_{i=1}^{j} s_{i j} \eta_{i} \quad(1 \leqq j \leqq n),
$$

each $s_{i j}$ being an element of $\mathcal{C}$. It follows that

$$
\sigma \delta_{k}\left(\eta_{j} / \eta_{1}\right)=\sum_{i=2}^{j} s_{11}^{-1} s_{i j} \delta_{k}\left(\eta_{i} / \eta_{1}\right) \quad(2 \leqq j \leqq n)
$$

for each $k$, from which it easily follows that $\mathcal{f}^{0}\left\langle\delta_{k}\left(\eta_{2} / \eta_{1}\right), \cdots\right.$, 
$\left.\delta_{k}\left(\eta_{n} / \eta_{1}\right)\right\rangle$ is a Picard-Vessiot extension of $\mathcal{F}^{0}$ for which the corresponding group of automorphisms is in triangular form and therefore solvable. Now if $n=1$ it is obvious that $G$ is a liouvillian extension of $\mathcal{F}^{0}$ and therefore of $\mathcal{F}$. Let $n>1$ and suppose that for all lower values of $n$ we know that the group's having a solvable component of the identity implies that the Picard-Vessiot extension is liouvillian. Then, for each $k, \mathcal{F}^{0}\left\langle\delta_{k}\left(\eta_{2} / \eta_{1}\right), \cdots, \delta_{k}\left(\eta_{n} / \eta_{1}\right)\right\rangle$ is a liouvillian extension of $\mathcal{F}^{0}$, so that $\mathcal{F}^{0}\left\langle\left(\delta_{k}\left(\eta_{j} / \eta_{1}\right)\right)_{1 \leqq k \leqq m, 2 \leqq j \leqq n}\right\rangle$ is a liouvillian extension of $\mathcal{F}^{0}$. Now $\sigma \eta_{1}=s_{11} \eta_{1}$, so that $\sigma\left(\eta_{1}^{-1} \delta_{k} \eta_{1}\right)=\eta_{1}^{-1} \delta_{k} \eta_{1}$ for all $\sigma \in \mathcal{S}^{0}$, whence $\eta_{1}^{-1} \delta_{k} \eta_{1} \in \mathcal{F}^{0}(1 \leqq k \leqq n)$. It follows that $G=\mathcal{F}^{0}\left\langle\eta_{1}, \cdots, \eta_{n}\right\rangle$ is a liouvillian extension of $\mathcal{F}^{0}$, and therefore of $\mathcal{F}$. Thus we have proved the following result.

Theorem 4. Let $G$ be a Picard-Vessiot extension of $\mathcal{F}$, and let (5) be the group of all automorphisms of $G$ over $\mathcal{F}$ (suitably identified with an algebraic matric group over $(\mathcal{C})$. If $G$ is contained in a liouvillian extension of $\mathcal{F}$, then the component of the identity $(50$ is solvable. Conversely, if $3^{\circ}$ is solvable, then $G$ is itself a liouvillian extension of $\mathcal{F}$.

It is easy to refine this theorem by introducing, as in the ordinary case (PV, $\$ \S 24,25)$, ten types of liouvillian extensions and ten types of algebraic matric groups.

Columbia University 